\title{
A study on the Risk factors for Gallbladder diseases in El-khoms Teaching Hospital, Libya
}

\author{
Ahmed Gomati ${ }^{1}$, Saleh Elafi ${ }^{1} \&$ Hamad Rafe ${ }^{2}$ \\ 1. Surgery Department, Faculty of Medicine, El-margeb University, El-khoms, Libya. \\ 2. Surgery Department, Faculty of Medicine, Omar Mukhtar University, Derna, Libya.
}

\begin{abstract}
Gallbladder disease (GBD) is one of the most common causes of hospital admission in the developed countries, resulting in a substantial cost to health system. The aim of this study is on demographic,

characteristic of GBD patients and the associated risk factors.

This case-control study includes 200 patients (cases) with GBD, randomly selected from surgery department at el-khoms teaching hospital, Libya from 1 October 2007 to 30 May 2008. In addition,200 subjects matched with age \& sex from other inpatients not suffering from GBD were chosen as controls. Ethical considerations were taken. A detailed history, physical examination, biochemical measurements and abdominal sonography were done. A well formed questionnaire for each subject was filled and full investigations were done.

Of all studied subjects (400), males were 240 (60\%) and females were 160 (40\%) with a mean age of 49.1 years (ranging from 18 to 70 yrs). The rate of GBD among inpatient cases during the study period were $26 \%$ with more predominance among females (f/m ratio 5.6:1). Risk factors for GBDs were: positive family history $(O R=$ 1.9, $P<0.05)$, multipara $(O R=2.9, P<0.05)$, use of oral contraceptive pills $(O R=2.98, P<0.046)$, hormone therapy $(O R=1.3, P<0.01)$, high $B M I(O R=1.3, P<0.05)$ and hyperlipidemia $(O R=6.3, P<0.01)$.

The rate of GBD was high. This was partly explained by unfavorable body mass index, lipid levels, higher prevalence of diabetes, and a more frequent use of oral contraceptives and hormone therapy in subjects.

Key words: Gallbladder diseases, Risk factors, Prevalence, El-khoms Teaching Hospital.
\end{abstract}

\section{Introduction}

Gallbladder disease is one of the most common causes of hospital admission in middle-aged patients in developed countries (Kang et al.,2003 and Kratzer et al., 1999) resulting in a substantial cost to health systems (Aerts and Penninckx, 2003).

Various numbers of factors including high age, female sex, over-weight, food pattern, and a sedentary lifestyle have been identified to be associated with a higher risk of gallstones (Volzke et al., 2005 and Shaffer, 2006).

Women are 1.5-3 times more frequently affected by gallstones than men. Pregnancies might be the main factor, but also the use of oral contraceptives (OCs) or menopausal hormone therapy (MHT) might play a role (Uhler et al., 2000; Thijs and Knipschild, 1993 and Dhiman and Chawla, 2006). In 31\% of pregnant women occurs biliary sludge (Maringhini et al., 1993), which is a potential mediator of gallstone formation. Moreover, use of $\mathrm{OC}$ and MHT causes an increase of cholesterol secretion, which promotes gallstone formation via cholesterol super saturation of bile (Mandez-Sanchez et al., 2007).

Supersaturated bile and increased hepatic secretion of cholesterol also possibly link to obesity and gallstones. A further determinate of gallstone formation is diet. A high intake of saturated fat and carbohydrate as well as a high glycemic load and index is associated with an increased gallstones rate, whereas a high fat protects against gall stone disease (Katsika et al., 2005).

\section{Aim of study}

This study intends to assess the risk factors associated with occurrence of gallbladder disease.

\section{Subjects and Methods}

This case-control study included 200 patients (cases) with GBD, randomly selected from surgery department at El-khoms teaching hospital, Libya from $1^{\text {st }}$ October, 2007 to $30^{\text {th }}$ May, 2008. In addition, 200 subjects matched with age and sex, from other inpatients not suffering from GBD was chosen as controls. Ethical considerations were taken. A detailed history, physical examination, biochemical measurements, and abdominal sonography were done. A well-formed questionnaire for each subject was filled and full investigation was done.

Patients were identified as having a hospital admission for gallbladder disease if they had a hospital record where the primary diagnosis was cholelithiasis or cholecystitis (ICD-10 codes K80-K81) or where any procedure code indicated a cholecystectomy (OPCS -4 codes J18). 
Body mass index (BMI) was calculated as $\mathrm{kg} / \mathrm{m}^{2}$ and categorized according to the WHO criteria (underweight: $>18.5 \mathrm{~kg} / \mathrm{m}^{2}$, normal: $18.5-24.99 \mathrm{~kg} / \mathrm{m}^{2}$, overweight: $25-29.99 \mathrm{~kg} / \mathrm{m}^{2}$, obese: $>30 \mathrm{~kg} / \mathrm{m}^{2}$ ) (WHO. 2000).

BMI was calculated using self-reported height and weight collected from the participants were categorized according to the following BMI categories: $<18.518 .5-24.9,25-29.9,30-39.9$ and $40+\mathrm{kg} / \mathrm{m}^{2}$.

Serum total cholesterol and HDL and LDL cholesterol levels were measured by enzymatic method (Hitachi 704, Roche, Mannheim, Germany).

\section{Statistical analyses}

Continuous data are expressed as means (SD). Nominal data are expressed as percentages. For bivariate analyses, the kruskal-wallis test (continuous data) or t-test (nominal data) was used.

\section{Results}

Table 1 shows that of all studied subjects (400), males were $240(60 \%)$ and females were $160 \quad(40 \%)$ with a mean age of 49.1 years (ranging from 18 to $70 \mathrm{yrs}$ ). There was more predominance among females (flm ratio 5.6:1).

Table 2 shows that risk factors for GBDs were: positive family history $(\mathrm{OR}=1.9, \mathrm{P}<0.05)$, multipara $(\mathrm{OR}=2.9$, $\mathrm{P}<0.05]$, use of oral contraceptive pills $(\mathrm{OR}=2.98, \mathrm{P}<0.046]$, hormone therapy $(\mathrm{OR}=1.3, \mathrm{P}<0.01]$, and hyperlipidemia $(\mathrm{OR}=6.3, \mathrm{P}<0.01]$.

Table 3 shows the distribution of studied subject according to body mass index (BMI).

\section{Discussion}

The factors adjusted for are well-accepted risk factors for gallstones. Fatness is a well-documented risk factor, especially in women, where the risk of gallstones increases linearly with rising body weight. Diabetes is also associated with gallstones. However, the association might be confounded by factors such as age and BMI (Jorgensen, 1989 and Maclure et al., 1989).

\section{Gallbladder Diseases with BMI and Hyperlipidemia:}

Risk factors for GBDs were high $\mathrm{BMI}(\mathrm{OR}=1.3, \mathrm{P}<0.05)$ and hyperlipidemia $(\mathrm{OR}=6.3, \mathrm{P}<0.01)$.

The role of serum lipids in the etiology of gallstones is not clearly understood. In general, it is assumed that hyperlipidemia is associated with gallstones. However, several studies found inverse associations of serum HDL, LDL, and total cholesterol with gallstones. Furthermore, a higher prevalence of gallstones among men on cholesterol-lowering diet was observed (Thijs et al., 1990 and Martinez et al., 1997).

\section{Gallbladder Diseases with Multipara and Oral Contraceptive:}

Risk factors for GBDs were multipara $(\mathrm{OR}=2.9, \mathrm{P}<0.05)$ and the use of Oral contraceptive pills $(\mathrm{OR}=2.98$, $\mathrm{P}<0.046$ ).

Whereas pregnancy is a well- established risk factor for gallstones, the effect of Oral Contraceptive (OC) and menopausal hormonal therapy (MHT) is still controversially discussed. Older studies found a higher risk of gallstones in women who had taken OC. However, the findings in other studies are inconsistent. The progressive reduction in hormone content and the incomparable doses and types of estrogen or progestin in used OC might be possible explanations for this lack of consistency. The underlying hypothesis assumes that the use of OR cause an increase of biliary cholesterol saturation (Kono et al., 1995; Cuevas et al., 2004 and Tsai et al., 2004). Moreover, family history of gallstones is a strong risk factor for gallstone disease. The evidence for a genetic component to gallstone disease has been grown during the last years (Tsai et al., 2005 and Buch et al., 2007).

\section{Gallbladder diseases with Positive family history:}

Risk factors for GBDs was a positive family history $(\mathrm{OR}=1.9, \mathrm{P}<0.05)$.

Gallstone formation is thought to rely on 3 factors: super saturation of biliary cholesterol due to hepatic hypersecretion, nucleation of cholesterol monohydrate crystals, and gallbladder hypo motility. The liver has estrogen receptors, and the presence of endogenous estrogens causes cholesterol saturation in the bile, inhibition of chenodeoxycholic acid secretion, and increased cholic acid content. Progestin inhibits gallbladder contraction, encourage bile stasis and have been shown to decrease the gallbladder's response to cholecystokinin. One study found that exogenous estrogens, given either transdermally or orally, affected physiologic markers in a pattern that favored gallstone formation (Donovan, 1999 and George and Schluger 2000).

\section{Gallbladder Diseases with Hormone therapy}

Risk factors for GBDS was hormone therapy $(\mathrm{OR}=1.3, \mathrm{P}<0.01)$.

Observational evidence suggests that estrogen therapy, including the use of $\mathrm{OC}$ and postmenopausal estrogen therapy, is an important risk factor for gallbladder disease. Observational studies indicate up to a 2.5 fold 
increased risk of biliary tract conditions related to estrogen therapy. A previous randomized trial examining biliary tract outcomes after estrogen therapy in postmenopausal women was the Heart and Estrogen\Progestin replacement study (HERS), which included women with known cardiovascular disease. HERS data revealed a $38 \%$ increased risk for biliary tract surgery in the estrogen therapy group. Thus, limited clinical trial evidence is available to provide unbiased estimates of the true effects and risks of estrogen therapy in postmenopausal women (Nelson et al., 2002 and Boland et al.,2002).

Estrogens appear to influence several key steps in gallstone formation. One study of postmenopausal women found that exogenous CEEs given orally or estradiol given transdermally, decreased nucleation time in vitro, increased cholesterol saturation index and increased biliary arachidonate and prostaglandin $\mathrm{E}_{2}$ levels. The pattern of findings resulted in both an increased propensity to form cholesterol crystals and an excess saturation of biliary cholesterol, which along with hypo motility of the gallbladder are thought to be key requirements for gallstone formation. The effect of estrogen therapy has also been studied in men who received exogenous estrogens. In an autopsy study of men randomized to estrogen therapy for the treatment of prostate cancer, the rate of Cholecystectomy was higher but the prevalence of Cholelithiasis among estrogen users were lower at time of death (Liu et al., 2008 and Williams et al., 2008).

\section{Conclusion}

The rate of GBD in this study was high. This was partly explained by unfavorable body mass index, lipid levels, higher prevalence of diabetes and a more frequent use of oral contraceptives and hormone therapy in subjects.

\section{References}

[1]. Aerts R., Penninckx F. (2003) The burden of gallstone disease in Europe. Aliment Pharmacol Ther, 18 (suppl 3):49-53.

[2]. Basso L., McCollum P.T. and Darling M.R. (1992) A descriptive study of pregnant women with gallstones. Relation to dietary and social habits, education, physical activity, height and weight, Eur j epidemiol 8:629-633.

[3]. Boland L.L., Folosm A.R. and Rosamond W.D. (2002) Atherosclerosis risk in communities study i; hyperinsulinemia, dyslipidemia, and obesity as risk factors for hospitalized gallbladder disease;a prospective study. Ann epidemiol. 12:131-140.

[4]. Buch S., Schafmayer C. and Volzke H. (2007) A genome -wide association scan identifies the hepatic cholesterol transporter ABCG8 as a susceptibility factor for human gallstone disease. Nat Genet 39:995-999.

[5]. Cuevas A., Miquel J.F. and Reyes M.S. (2004) Diet as a risk factor for cholesterol gallstone disease. J am coll Nutr 23:187-196.

[6]. Dhiman R.K. and Chawla Y.K. (2006) Is there a link between estrogen therapy and gallbladder disease? Expert Opin Drug Saf 5:117-129.

[7]. Donovan J.M. (1999) Physical and metabolic factors in gallstone pathogenesis. Gastroenterol Clin North Am.28:75-97.

[8]. George E.D. and Schluger L.k. (2000) Special women's health issues in hepatobiliary disease. Clinics in Family Practice.2:155169.

[9]. Jorgensen T. (1989) Gall stones in a Danish population. Relation to weight, physical activity, smoking, coffee consumption and diabetes mellitus. Gut 30:528-534.

[10]. Kang J.Y., Ellis C. and Majeed A. (2003) Gallstones- an increasing problem: a study of hospital admissions in England between 1989\1990 and 1999\2000 Aliment Pharmacol Ther 17:561-569.

[11]. Katsika D., Grjibovski A. and Einarsson C. (2005) Genetic and environmental influences on symptomatic gallstone disease: a Swedish study of 43,141 twin pairs. Hepatology 41:1138-1143

[12]. Kono S., Shinchi K. and Todoroki I. (1995) Gallstones disease among Japanese men in relation to obesity, glucose intolerance, exercise, alcohol use and smoking. Scand j Gastroenterol 30:372-376.

[13]. Kratzer W., Mason R.A. and Kachele V. (1999) Prevalence of gallstones in sonographic surveys worldwide. J Clin Ultrasound 27:17.

[14]. Liu B., Beral V., Balkwill A., Green J., sweetland S. and Reeves G. (2008) Gallbladder disease and use of transdermal versus oral hormone replacement therapy in postmenopausal women .BMJ.337:386. Doi:10.1136/BMJ.a 386.

[15]. Maclur K.M., Hayesk C. and Colditz G.A. (1989) Weight, diet, and the risk of symptomatic gallstones in middle-aged women. N Engl J Med 321:563-569.

[16]. Maringhini A., Ciambra M. and Baccelliere P. (1993) Biliary sludge and gallstones in pregnancy: Incidence, risk factors, and natural history. Ann Intern Med 119:116-120

[17]. Martinez de pancorbo C., Carballo F. and Horcajo P. (1997) Prevalence and associated factors for gallstone disease: results of a population survey in Spain. J Clin Epidemiol 50:1347-1355

[18]. Mendez-sanchez N., Zamora-valdes D. and Chavez-tapian C. (2007) et al. Role of diet in cholesterol gallstone formation. Clin Chim Acta 376:1-8.

[19]. Nelson H.D., Humphrey L.L., Nygren P., Teutsch S.M. and Allan J.D. (2002) Postmenopausal hormone replacement therapy: scientific review. Jama. 288:872-881.

[20]. Shaffer E.A. (2006) Gallstone disease: Epidemiology of gallbladder stone disease. Best Pract Res Clin Gastroenterol 20:981-996

[21]. Thijs C., Knipschild P. and Brobacher P. (1990) Serum lipids and gallstones: a case-control study. Gastroenterology 99:843-849.

[22]. Thijs C. and Knipschild P. (1993) Oral contraceptives and the risk of gallbladder disease;ameta analysis. Am J Public Health 83:1113-1120.

[23]. Tsai C.J., Leitzmann M.F. and Willet W.C. (2004) The effect of long-term intake of cis unsaturated fats on the risk for gallstones disease in men: a prospective cohort study. Ann Intern Med 141:514-522.

[24]. TsaiC.J., Leitzmann M.F. and Willett W.C. (2005) Dietary carbohydrates and glycaemic load and the incidence of symptomatic gall stone disease in men. Gut 54:823-828.

[25]. Uhler M.L., Marks J.W. and Judd H.L. (2000) Estrogen replacement therapy and gallbladder disease on postmenopausal women. Menopause 7:162-167.

[26]. Volzke H., Baumeister S.E. and Alte D. (2005) Independent risk factors for gallstone formation in a region with high cholelithiasis prevalence. Digestion 71:97-105. 
[27]. W.H.O. (2000) Obesity: preventing and managing the global epidemic. Report of a who consultation. WHO technical report series 894. World Health Organization: Geneva.

[28]. Williams E.J., Green J. and Beckingham I. (2008) Guidelines on the management of common bile duct stones (CBDS). Gut. 57 (7):1004-1021.

Table 1: Age and sex distribution of studied subject:

\begin{tabular}{|c|c|c|c|c|}
\hline \multicolumn{2}{|c|}{ Age Groups } & $\mathbf{0 \%}$ & \multicolumn{2}{c|}{ Female } \\
\hline $10-$ & 0 & 0 & 4 & 2.4 \\
\hline $20-$ & 2 & 6.7 & 58 & 22.4 \\
\hline $30-$ & 2 & 6.7 & 26 & 29.4 \\
\hline $40-$ & 0 & 0 & 30 & 15.3 \\
\hline $50-$ & 14 & 46.6 & 22 & 17.6 \\
\hline $60+$ & 12 & 40 & 170 & 12.9 \\
\hline Total & 30 & 100 & & 100 \\
\hline
\end{tabular}

Table 2: Risk factors in studied subject:

\begin{tabular}{|l|c|c|c|c|}
\hline \multicolumn{1}{|c|}{ Risk Factor } & Patient & Controls & O.R. & P- value \\
\hline Diabetes mellitus & 20 & 40 & 0.44 & $>0.05$ \\
\hline Hypertension & 18 & 50 & 0.26 & $>0.05$ \\
\hline Hemolytic anemia & 4 & 2.02 & 0.03 \\
\hline Ischemic heart & 2 & 12 & 0.158 & $<0.05$ \\
\hline Positive family history & 80 & 52 & 2.93 & $<0.05$ \\
\hline Multipara & 136 & 84 & 2.98 & $<0.045$ \\
\hline Contraceptive pills & 32 & 12 & 6.31 & $<0.01$ \\
\hline Hormonal therapy & 14 & 2 & 1.0 & $<0.01$ \\
\hline High cholesterol & 12 & 24 & & $<0.001$ \\
\hline High triglyceride & 24 & & & \\
\hline
\end{tabular}

Table 3: Distribution of studied subject according to body mass index:

\begin{tabular}{|c|c|c|c|}
\hline BMI & Patient & Controls & O.R \\
\hline $19-$ & 2 & 2 & 1 \\
\hline $20-$ & 42 & 52 & 0.756 \\
\hline $25-$ & 68 & 64 & 1.080 \\
\hline $30-$ & 52 & 42 & 1.320 \\
\hline $35-$ & 14 & 26 & 0.910 \\
\hline $40+$ & 12 & 14 & 0.840 \\
\hline
\end{tabular}

